\title{
Guest Editorial for Special Issue on 3D Printing: Opportunities and Applications for Supply Chain Management
}

As ever more firms are geographically separating design, marketing and manufacturing, the role and importance of supply chain management has become central as an integration and coordination mechanism. Equally, this internationalisation of supply chains and networks has brought with it increased complexity and vulnerability to uncertainties. The rise of 3D Printing (3DP), also known as additive manufacturing technologies, provides firms with the opportunity of reconfiguring supply chains by bringing manufacturing closer to home and hence shortening or virtually eliminating lead times (Berman, 2012; Mellor et al., 2014; Weller et al., 2015). This allows for unprecedented co-creation and customisation/personalisation of products, leading to greater agility and responsiveness to customer needs. Other benefits include providing employment opportunities locally in a firm's home region, as well as establishing strategic delivery hubs.

Existing applications of 3D printing are already far reaching and include: precision parts for the automotive and aerospace industries, dental/bone implants and prosthetics, architectural visualisation models, print on demand personalised consumer durables (e.g. sport shoes, figurines, furniture, and jewellery) and spare parts. To date, 3D printed products have primarily been prototypes and low volume one-off products but as the technology develops and becomes feasible for larger volume manufacturing, the implications for supply chain configurations and coordination approaches are also becoming clearer (Holmström and Partanen, 2014; Sasson and Johnson, 2016).

Our special issue on "3D Printing: Opportunities and Applications for Supply Chain Management" aimed to extend understanding of the impact of 3DP on supply chain management. The call for papers was made public in the summer of 2016, with a submission deadline set for the end of 2016. Of the 14 papers submitted, 12 were subjected to a blind peer review process, which eventually led to the selection of the 3 papers presented in this issue.

The first paper included in our special issue, by Durach, Kurpjuweit and Wagner, , and, is titled "The Impact of Additive Manufacturing on Supply Chains". The authors began from the premise that there is a need for more empirical research to explore the impact of Additive Manufacturing (AM) on supply chains and based their research on the principle that different AM technologies employed will, potentially, have different implications for supply chains. Articulated by the authors in three research questions, the paper explores the current state and future potential of AM processes. Durach et al.'s research paper goes on to identify barriers for adoption of these technologies, indicating approximate timeframes to overcome them, and continues by exploring the impact on supply chains, again with indicative timeframes. Their literature review follows a structure relevant to the research questions, providing a critical review of the seven current major AM processes, and summarising the most dominant current barriers under four main categories and emphasising the limited current knowledge in terms of supply chain implications. This review of past research sets the basis for the two-stage surveybased empirical study that involved both practitioners and academics.

Durach et al.s' study identifies five leading AM processes as of now and potentially in the future, and then discusses their relative potential. Subsequently, the authors identify and discuss the implications of key barriers preventing widespread adoption of AM processes. Interestingly, the study findings contradict some of the past research in terms of the importance 
of commonly referred to barriers. Durach et al. also identify a set of "soft factor" barriers that they contend are both significant and under-researched. The analysis provides a detailed insight into potential impacts of the barriers on AM application, as well as an estimation for when these barriers may be overcome. Finally, the supply chain implications are identified under four categories, and discussed based on their likelihood and estimated time. Finally, in relation to the supply chain implications, the authors summarise their key findings in the form of eight propositions.

The second paper by Srivastava, Dwivedi and Srivastava, is titled "Analysis of Barriers to Implement Additive Manufacturing Technology in the Indian Automotive Sector: A FuzzyISM Approach". This paper has a specific industrial and geographical focus, aiming to examine barriers to the implementation of AM in the Indian automotive sector and to identify the interrelationships between these barriers. The authors initially briefly identify the benefits of $\mathrm{AM}$ and justify their selection of the Indian automotive sector as an appropriate context, together with the need to understand the barriers for AM implementation. The literature review explores in detail potential benefits and barriers of AM in the automotive sector. The study collected data from fifteen top and middle-level managers in two major Indian automotive companies, with both being market leaders in the passenger and commercial vehicles segments respectively. The modified Fuzzy Interpretive Structural Modelling (Fuzzy ISM) method employed allows the identification of the interrelations among the barriers. The study identifies, clusters and ranks the barriers to AM implementation, demonstrating how the barriers drive one another. The authors provide Initial and Final Fuzzy Reachability Matrices for indirect strength calculations, and an ISM diagraph to demonstrate the direct strength of the relationships. The barriers are grouped into four clusters based on driving power and dependence, which the authors rank in terms of criticality. This allows the relative delineation of these barriers, with academic and practical implications for other industries

The third paper, by Ryan, Eyers, Potter, Purvis andGosling, is titled "3D Printing the Future: Scenarios for Supply Chains Reviewed". The authors review existing 3DP scenarios and identify gaps, which they refer to as "white space" in current understanding of future 3DP scenarios and research opportunities. The paper initially scopes and outlines the benefit of scenario planning and development for supply chain management, and justifies the need to consolidate and review 3DP scenarios for their impact on supply chains. Ryan et al. developed a coding framework for the review of the scenarios focusing on three criteria: customer engagement, the geographic distribution of manufacturing, and the type of manufacturing operation. The authors sought out scenarios from academic publications, as well as in the popular press and industry publications. The publications collected underwent a systematic refining process to confirm their relevance, and the resulting structured literature review of 128 publications led to the identification of 201 scenarios. The authors provide a brief historical perspective based on the number of 3DP scenarios proposed and associated recent technological developments, and discuss the number of the publications that address the scenario effects and timescales. The discussion that follows builds upon the analysis of the scenarios and leads to the identification of five areas of "white space", i.e. where limited or no scenarios exist. The paper closes by proposing future research opportunities for 3DP supply chain development.

While the three papers explore 3D operations from different perspectives, the two major themes underlying them are 'barriers to 3D printing operationalisation' and 'future potential'; the latter being relevant to both 'timelines', as well as 'performance objectives'. We believe these are pertinent issues, and when complemented by Rogers et al.'s (2016) “3D printing services: 
Classification, supply chain implications and research agenda", an interesting pattern emerges. This pattern can best be conceptualised and represented diagrammatically as a 3DP continuous performance improvement cycle as shown in Figure 1. Supply chain strategy, structure and operations can be visualised as a 'dynamic' continuous improvement cycle which will continue to progress on an upward trajectory as the 'barriers' highlighted by in this special issue by Durach et al.'s paper are addressed, deploying techniques such as fuzzy Interpretive Structural Modelling (Fuzzy-ISM) proposed in Srivastava et al.'s contribution. (We believe that the proposed continuous improvement cycle encapsulating three pillars; namely strategy-structureoperations (SSO) of 3DP supply chains will continue to gather pace and move on an upwards trajectory, following the future scenarios proposed by the Ryan et al.'s paper. However, during the course of this trajectory the three pillars (SSO) will require regular recalibration to reconfigure and re-align 3DP supply chains as the demand for 3DP goods and services increases with time. Accordingly, the challenges for reconfiguring and performance of supply chains will increase over time. Hence the reason for the growing size of the continuous improvement cycle is represented as 'dashed' (rather than solid) lines in the second and third cycles of Figure 1.

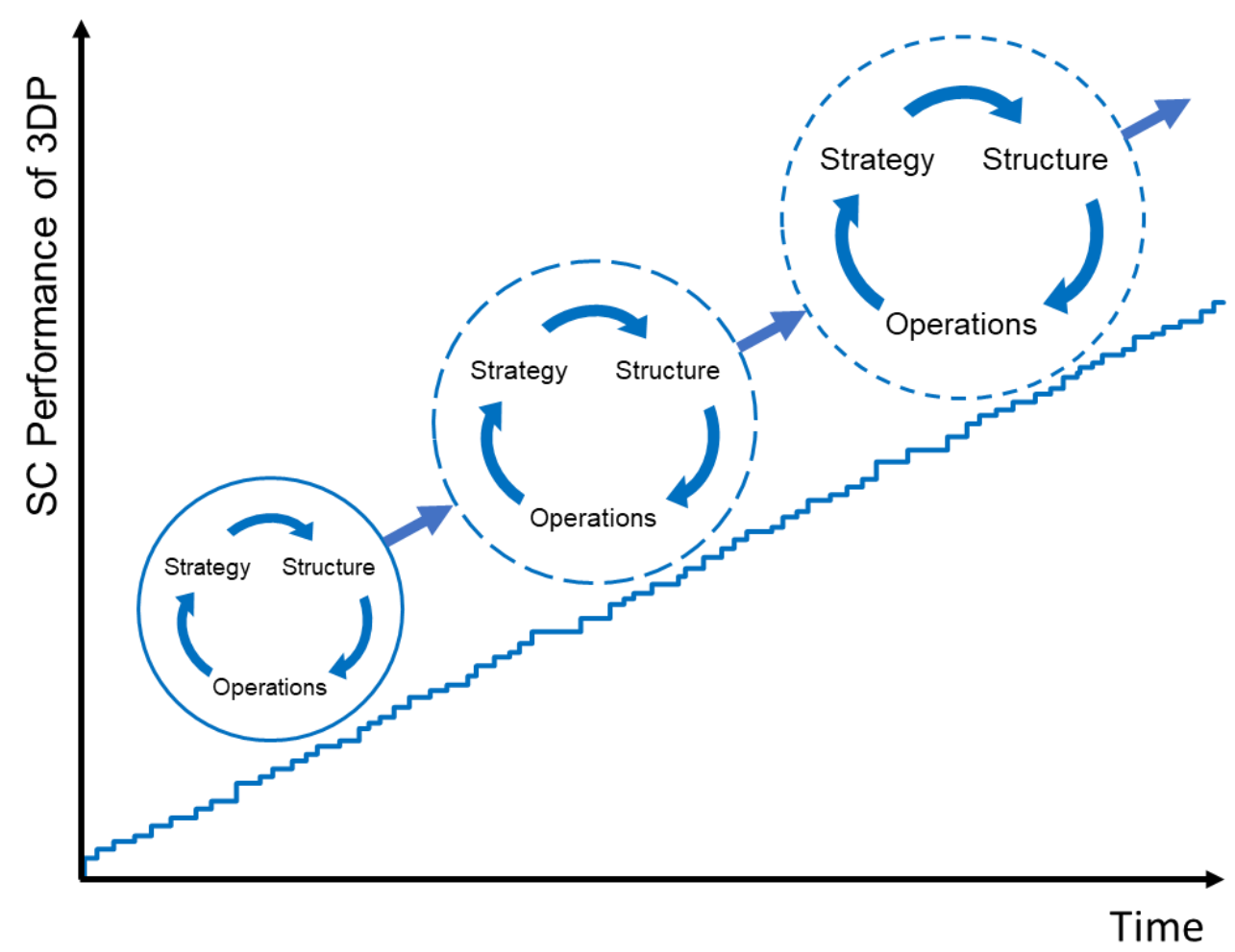

Figure 1: 3DP supply chain continuous performance improvement cycle

Reflecting back to the 1990s on the early days of the commercialisation of rapid prototyping (that would ultimately develop into AM/3DP), a key imperative for this special issue is to act as a catalyst for recapturing the activity and enthusiasm for the field that existed at this time. Initiatives such as this special issue can serve to mobilise much-needed 3DP supply chainrelated research projects and, in turn, may encourage constructive debate at regional, national and international levels amongst researchers, scholars and practitioners. 
Guest Editors:

Helen Rogers, Professor of International Business, Nuremberg Institute of Technology, Germany. helen.rogers@th-nuernberg.de

Christos Braziotis, Lecturer in Supply Chain and Operations Management, Nottingham University Business School, UK. Christos.Braziotis@ nottingham.ac.uk

Kulwant S Pawar, Professor of Operations Management, Nottingham University Business School, China and UK. Kul.Pawar@nottingham.ac.uk; Kulwant.Pawar@nottingham.edu.cn

\section{References}

Berman, B. (2012), “3-D printing: The new industrial revolution”, Business Horizons, Vol. 55 No. 2, pp. 155-162.

Holmström, J. and Partanen, J. (2014), "Digital manufacturing-driven transformations of service supply chains for complex products", Supply Chain Management: An International Journal, Vol. 19 No. 4, pp. 421-430.

Mellor, S., Hao, L. and Zhang, D. (2014), "Additive manufacturing: A framework for implementation", International Journal of Production Economics, Vol. 149, pp. 194201.

Rogers, H., Baricz, N. and Pawar, K.S. (2016), “3D printing services: Classification, supply chain implications and research agenda", International Journal of Physical Distribution \& Logistics Management, Vol. 46 No. 10, pp. 886-907.

Sasson, A. and Johnson, J.C. (2016), "The 3D printing order: Variability, supercenters and supply chain reconfigurations", International Journal of Physical Distribution \& Logistics Management, Vol. 46 No. 1, pp. 82-94.

Weller, C., Kleer, R. and Piller, F.T. (2015), "Economic implications of 3D printing: Market structure models in light of additive manufacturing revisited", International Journal of Production Economics, Vol. 164, pp. 43-56. 